\title{
Pediatric Issues in Times of Pandemia: From Infection to Nutritional Strategies
}

\section{Raanan Shamir ${ }^{a, b}$}

a Institute of Gastroenterology, Nutrition and Liver Diseases, Schneider Children's Medical Center, Petah Tikva, Israel; b Professor of Pediatrics, Lea and Arieh Pickel Chair for Pediatric Research, Sackler Faculty of Medicine, Tel-Aviv University, Tel Aviv, Israel

In December 2019, an outbreak of a new transmitted disease (COVID-19) was identified in Wuhan, China, caused by a new coronavirus (SARS-CoV-2), spreading quickly around the world and causing a pandemia that touched the lives of everyone, from pediatric to geriatric age [1-4]. In face of the pandemia, we decided to dedicate this issue of Annals of Nutrition and Metabolism to "Pediatric Issues in Times of Pandemia: From Infection to Nutritional Strategies". As immunity and nutrition have always been interrelated, we start this issue with a review on the relationship between long-chain polyunsaturated fatty acids (LCPUFA) and respiratory infections. This review is followed by the challenges of breastfeeding and tackling protein-calorie malnutrition during the pandemia as two out of many nutritional challenges brought by this crisis.

The first paper in this issue deals with a specific group of nutrients, LCPUFAs, as an example for the relationship between nutrition, immunity, and infection [5]. Over many decades, the role of LCPUFAs has attracted the interest of the medical and research community, of the media and of policymakers. However, the role of LCPUFAs in preventing and attenuating the course of respiratory viral infections has received limited attention. In their review, the authors explore randomized controlled trials performed in both pediatric and adult subjects, evaluating the protective effect of LCPUFA supplementation against respiratory tract infections. The authors reiterate the importance of LCPUFAs and LCPUFA metabolites in acute and chronic inflammation and point out the need to study the role of LCPUFAs in COVID-19, as well as the difficulty in reaching conclusions in nutrition intervention studies due to low quality of evidence and lack of studies evaluating these interventions as a primary outcome.

In the second paper, the authors provide a valuable review on breastfeeding during the COVID-19 pandemia [6]. This is a comprehensive review, highlighting many aspects of breastfeeding, challenges involved as well as remaining controversies. As the COVID-19 pandemia contributed to breastfeeding cessation and insecurity, the review emphasizes the need to employ strategies to support breastfeeding during the pandemia and to maintain it while employing strict hygiene measures. Interestingly, the review regards the newborn baby and his/her mother as a vulnerable population targeted by this world crisis and reiterates the need to maintain what we know about breastfeeding, namely that it is the gold standard for infant feeding and the recommended way to exclusively feed the infant in the first 6 months of life (ESPGHAN), and the difficulties in doing it in a rapidly changing world.

Following the review on the optimal way to feed infants also in times of a pandemia, and observing the huge societal 
challenge of malnutrition during a pandemia, we elected to close this issue with a review that summarizes the effects of the COVID-19 pandemia on malnutrition [7]. The world was not coping well with malnutrition long before COVID-19, and the pandemia met a world with a large number of malnourished adults and children. In the year 2020, globally, among children under the age of 5 years, about 45 million were estimated to be wasted, 149 million were stunted, and 38.9 million were estimated to be overweight or obese [8]. The authors provide an overview of causes for the increase in malnutrition burden and its societal and economic consequences and highlight national initiatives aimed at supporting disadvantaged groups, providing suggested action items to improving nutritional outcomes in these populations.

\section{References}

1 Spolidoro GC, Azzolino D, Shamir R, Cesari M, Agostoni C. Joint Effort towards Preventing Nutritional Deficiencies at the Extremes of Life during COVID-19. Nutrients. 2021 May;13(5):1616.

2 Li Q, Guan X, Wu P, Wang X, Zhou L, Tong Y, et al. Early Transmission Dynamics in Wuhan, China, of Novel Coronavirus-Infected Pneumonia. N Engl J Med. 2020 Mar;382(13):1199-207.

3 Murray KF, Gold BD, Shamir R, Agostoni C, Pierre-Alvarez R, Kolacek S, et al. Coronavirus Disease 2019 and the Pediatric Gastroenterologist. J Pediatr Gastroenterol Nutr. 2020 Jun;70(6):7206.

4 Phelan AL, Katz R, Gostin LO. The Novel Coronavirus Originating in Wuhan, China: Challenges for Global Health Governance. JAMA. 2020 Feb;323(8):709-10.
We hope that our readers will find these reviews educating and, hopefully, they will stimulate further research to improve the health of children worldwide during and after the pandemia.

\section{Conflict of Interest Statement}

The writing of this article was supported by Nestlé Nutrition Institute. The author has participated as a clinical investigator, and/or advisory board member, and/or consultant, and/or speaker for Abbott, Else, Nestlé Nutrition Institute, NGS, Nutricia, Soremartec, and Ukko.

\section{Funding Sources}

The author received no funding.

5 De Cosmi V, Mazzocchi A, Turolo S, Syren ML, Milani GP, Agostoni C. Long-chain polyunsaturated fatty acid supplementation and respiratory infections. Ann Nutr Metab. 2022, doi: 10.1159/000522093.

6 Milani GP, Porro A, Agostoni C, Giannì ML. Breastfeeding during a pandemic. Ann Nutr Metab. 2022, doi: 10.1159/000521349.

7 Padhani ZA, Das JK, Akhtar S, Ismail T, Bhutta ZA. Tackling proteincalorie malnutrition during world crises. Ann Nutr Metab. 2022, doi: $10.1159 / 000522242$.

8 World Health Organization. Malnutrition: Factsheet. 2021 [cited 2021 Oct 22]. Available from: https://www.who.int/news-room/ fact-sheets/detail/malnutrition. 